\title{
The Effects of Bilirubin on Evoked Potentials and Long-Term Potentiation in Rat Hippocampus In Vivo
}

\author{
LIAN ZHANG, WANJUN LIU, A. KEITH TANSWELL, AND XIAOPING LUO \\ Department of Pediatrics [L.Z., W.L., X.L.], Tongji Hospital, Tongji Medical College, Huazhong \\ University of Science and Technology, Wuhan 430030, P.R. China; Hospital for Sick Children Research \\ Institute [A.K.T., X.L.], and the Department of Paediatrics, Faculty of Medicine, University of Toronto, \\ Toronto, Ontario M5G $1 X 8$, Canada
}

\begin{abstract}
ABSTR
Neonatal jaundice is a common condition that could poten-
tially lead to severe neurotoxicity. In this electrophysiological
study we observed the effects of a short-term bilirubin injection
on evoked potentials (population spike, PS) and long-term po-
tentiation (LTP) in the hippocampal CA $\mathrm{CA}_{3}$ region of Sprague
Dawley rats in vivo. The animal received a bolus i.v. injection of
either $60 \mathrm{mg} / \mathrm{kg}$, or $30 \mathrm{mg} / \mathrm{kg}$ of bilirubin, or an equivalent
volume of bilirubin-free vehicle in 5 min. The results showed
that both bilirubin-treated groups had a dose-independent pro-
longation of peak latencies and decrease of slopes of the PS at all
measured time points following injection (1, 3, 5 , 10, 15, 30, 45 ,
$60,90,120$ min), while the amplitudes of the PS did not change
significantly. The peak latency, slope, and the amplitude of PS
stayed unchanged in the control group. Furthermore, while LTP
could be induced by high-frequency stimulation in control ani-
mals, this phenomenon was absent in both bilirubin-treated
\end{abstract}
Hyperbilirubinemia is a common phenomenon in the neonatal period, and attracts a lot of attention due to its potential for neurotoxicity (1). Recent studies have shown that bilirubin may exert effects on neurons via multiple mechanisms including inhibition of protein phosphorylation (2) and release of neurotransmitters (3). However, these studies were carried out in vitro, and the results provide limited information on the sustained effects of bilirubin in the body.

Long-term potentiation (LTP) was first documented in the dentate gyrus of rabbit hippocampi by Bliss et al. (4). They stimulated the perforant path (PP) at each observed time point by high-frequency electricity with a given frequency and strength. Following this, single stimulation would significantly and last-

Received December 6, 2001; accepted June 4, 2002.

Correspondence: Department of Pediatrics, Tongji Hospital, Tongji Medical College, Huazhong University of Science and Technology, Wuhan 430030, P.R. China; e-mail: zhliancn@hotmail.com

Supported by the National Science Fund for Distinguished Young Scholars (Dr. Xiaoping Luo) from the Natural Science Foundation of China (Grant No. 30125019).

DOI: 10.1203/01.PDR.0000061563.63230.86 groups. The amplitudes of the PS in the two treated groups after stimulation were also smaller than those of the control animals at every time points. These findings are in accordance with previous observations showing significant depressive effects of bilirubin on the nervous system. Our novel finding that short-term exposure to bilirubin can inhibit the induction of LTPs in the hippocampus, is compatible with the suggestion that neonatal hyperbilirubinemia can impact on learning and memory. (Pediatr Res 53: 939-944, 2003)
LTP, Long-term potentiation
PP, perforant path
pEPSP, population excitatory postsynaptic potential
PS, population spike

ingly increase the PS in the dentate gyrus as well as the so-called population excitatory postsynaptic potential (pEPSP). This phenomenon was called LTP and has also been observed in other parts of hippocampus. LTP in the hippocampus is now considered as an important parameter related to learning and memory (5).

An investigation of the correlation between bilirubin and LTP might contribute to elucidating the mechanism of bilirubin neurotoxicity and perhaps in understanding the long-term effects of bilirubin encephalopathy. We hypothesized that bilirubin neurotoxicity may affect individual neuronal functions in distinctive ways and with variable degrees of reversibility. In this in vivo electrophysiological study, we observed the effects of an i.v. bilirubin bolus on the evoked potential (population spike, PS) and the LTP in the hippocampal $\mathrm{CA}_{3}$ region of Sprague Dawley rats.

\section{MATERIALS AND METHODS}

Crystalline bilirubin was purchased from Sigma Chemical Co., St. Louis, MO, U.S.A. Other reagents were analytical 
grade chemicals and were obtained from regular commercial sources. Sprague Dawley rats were supplied by the Center for Experimental Animals, Tongji Medical College.

Bilirubin solution. Crystalline bilirubin was dissolved in 0.1 $\mathrm{N} \mathrm{NaOH}$, and BSA was added to a molecular ratio of bilirubin:albumin $(\mathrm{B} / \mathrm{A}=14)$. This was further diluted in $0.055 \mathrm{M}$ phosphate buffer $(\mathrm{pH}=7.4)$ to a final bilirubin concentration of $3 \mathrm{mg} / \mathrm{mL}$. The control solution was identical except for the use of bilirubin. The whole process was accomplished under red light condition.

Animal experiments. Male or female Sprague Dawley rats, weighing 200-250 g, were anesthetized with $1 \mathrm{~g} / \mathrm{kg}$ of Urethane i.p. The trachea and cervical veins were cannulated and the scalps were incised. The rats were positioned prone and the heads were fixed on a SN-3 Brain Space Positioner (Narishige, Japan), with the anterior fontanel $1 \mathrm{~mm}$ higher than the posterior fontanel. The bipolar stimulating electrodes were placed on the perforant path (PP, 6.9-7.0 $\mathrm{mm}$ behind the anterior fontanel, 4.3-4.4mm right or left laterally, 5.0-5.3 $\mathrm{mm}$ in depth), according to the method of Bliss et al. (4). The perforant path was stimulated with single electric pulses (testing stimuli, range $0.15 \mathrm{~ms}$, strength $30 \mathrm{~V}$ ). The recording electrodes were placed in the hippocampal $\mathrm{CA}_{3}$ region $(3.3-3.4 \mathrm{~mm}$ behind the anterior fontanel, 3.3-3.5 mm right or left lateral, 3.5-3.8 $\mathrm{mm}$ in depth) and the evoked population spikes (PS) were recorded. The peak latency of PS was considered as the delay between onset and peak of PS.

When investigating the relationship between bilirubin and LTP, trains of high frequency stimuli (frequency $500 \mathrm{~Hz}$, range $0.15 \mathrm{~ms}$, strength $30 \mathrm{~V}$, duration $100 \mathrm{~ms} \times 2$ ) were given at the perforant path after the PS was detected, changes in the PS were then recorded. All signals were enlarged by a directcurrent amplifier and monitored on an oscilloscope, connected to an IBM computer. The main function of the computer is to collect and average responses, to measure various parameters of the responses, and to store and plot information.

The experiments were carried out in two steps. In part I, the animals were randomly divided into three groups (control, low-dose and high-dose, with 6 rats in each group) after the PS was recorded. $5 \mathrm{~min}$ later PS was recorded again and bilirubin $0,30 \mathrm{mg} / \mathrm{kg}$, or $60 \mathrm{mg} / \mathrm{kg}$, respectively, was injected as a $5-\mathrm{min}$ i.v. bolus. The solutions were given as the following: control group (20 mL/kg of control solution), the low dose group (10 $\mathrm{mL} / \mathrm{kg}$ of original bilirubin solution plus $10 \mathrm{~mL} / \mathrm{kg}$ of control solution), the high dose group (60 $\mathrm{mg} / \mathrm{kg}$ of bilirubin, 20 $\mathrm{mL} / \mathrm{kg}$ of original bilirubin solution). The final loading volume for the three groups was identical. The amplitude, slope and peak latency of the PS were collected at 1, 3, 5, 10, 15, 30, 45, 60,90 , and $120 \mathrm{~min}$ after injection. Injections were carried out under red light conditions with the animals breathing spontaneously. In part II, the effect of bilirubin on the induction of LTP was determined. The same method was used to make control, low-dose and high-dose groups after the PS had been measured. High frequency stimuli were given 5 min after the injection. The amplitudes of PS were recorded at 1, 3, 5, 10, 15, 30, 45, 60, 90, and $120 \mathrm{~min}$ after injection.

All procedures involving animals were conducted according to criteria established by the Huazhong University of Science and Technology and prior approval was obtained from the Animal Care Review Committee at the Tongji Hospital.

Statistical analysis. All numerical data are presented as mean \pm SEM. Statistical significance $(p<0.05)$ was determined by ANOVA.

\section{RESULTS}

\section{The Effect of Bilirubin on PS in the Hippocampal $\mathrm{CA}_{3}$ Region}

Effect of bilirubin on PS amplitude. PS amplitudes were recorded by computer at all observed time points. The normalized PS amplitude was presented as the ratio of PS amplitude $5 \mathrm{~min}$ before and every time points after treatment, as illustrated in Fig. 1. Statistical analysis showed no significant difference before and after treatment in each group. We could not detect significant differences between the control and the bilirubin treated groups. These results suggest that the concentration of bilirubin used in our experiment had no effect on PS amplitude during the observed time period.

Effect of bilirubin on PS peak latency. Changes of PS peak latencies of all treated groups were shown in Fig. 2. Prolonged PS peak latencies were evident after low- or high-dose bilirubin treatment at all observed time points $(p<0.05)$, while the control group did not change. Both low- and high-dose bilirubin treated groups had longer PS peak latencies compared with the control group $(p<0.05)$. However, there were no significant differences between the low- and high-dose bilirubin treated groups at each time point. This suggested that bilirubininduced prolongation of PS peak latency could last for up to $2 \mathrm{~h}$.

Effects of bilirubin on PS slope. As showed in Fig. 3, both treated groups showed the significant decrease of normalized PS slope $(p<0.05)$ at all observed time points after bilirubin application. But no statistically significant differences were found between the two groups. The normalized PS slope of the control group stayed unchanged during the whole process. Like the changes of peak latency, the reduction also lasted for $2 \mathrm{~h}$.

\section{The Effect of Bilirubin on the Induction of LTP in Rat Hippocampal $\mathrm{CA}_{3}$ Region}

LTP formation. To induce LTP, we first recorded a PS in the $\mathrm{CA}_{3}$ region after stimulating the perforant path by single electric pulses (strength $30 \mathrm{~V}$, range $0.15 \mathrm{~ms}$ ), and then applied high frequency stimuli $(500 \mathrm{~Hz}, 30 \mathrm{~V}$, range $0.15 \mathrm{~ms}$, duration $100 \mathrm{~ms} \times 2$ ) when the above-mentioned PS became stable. If the PS amplitude was significantly increased for more than 30 min, induction of LTP was confirmed.

Effect of bilirubin on LTP induction. Solvent, low- or high-doses of bilirubin were administered to the rats after stable PSs in the $\mathrm{CA}_{3}$ region had been recorded. Following high-frequency electric stimuli, the evoked PSs were measured and analyzed by computer. The normalized PS amplitude of the three groups are presented in Fig. 4. The PS amplitude of the control group was significantly increased after stimulation ( $p<0.01)$, indicating that LTP was formed. No significant increases for the PS amplitude were recorded in the two 

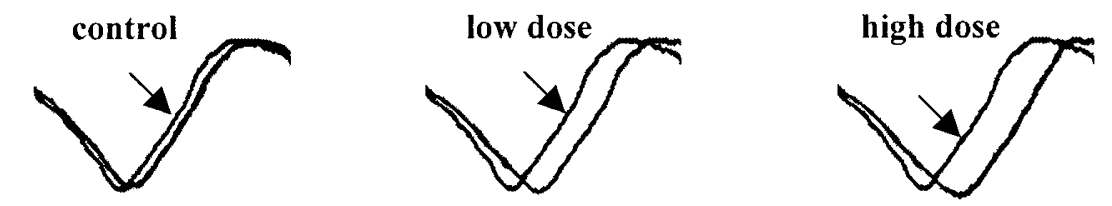

\section{$200 \mathrm{mv}$ \\ $2 \mathrm{~ms}$}

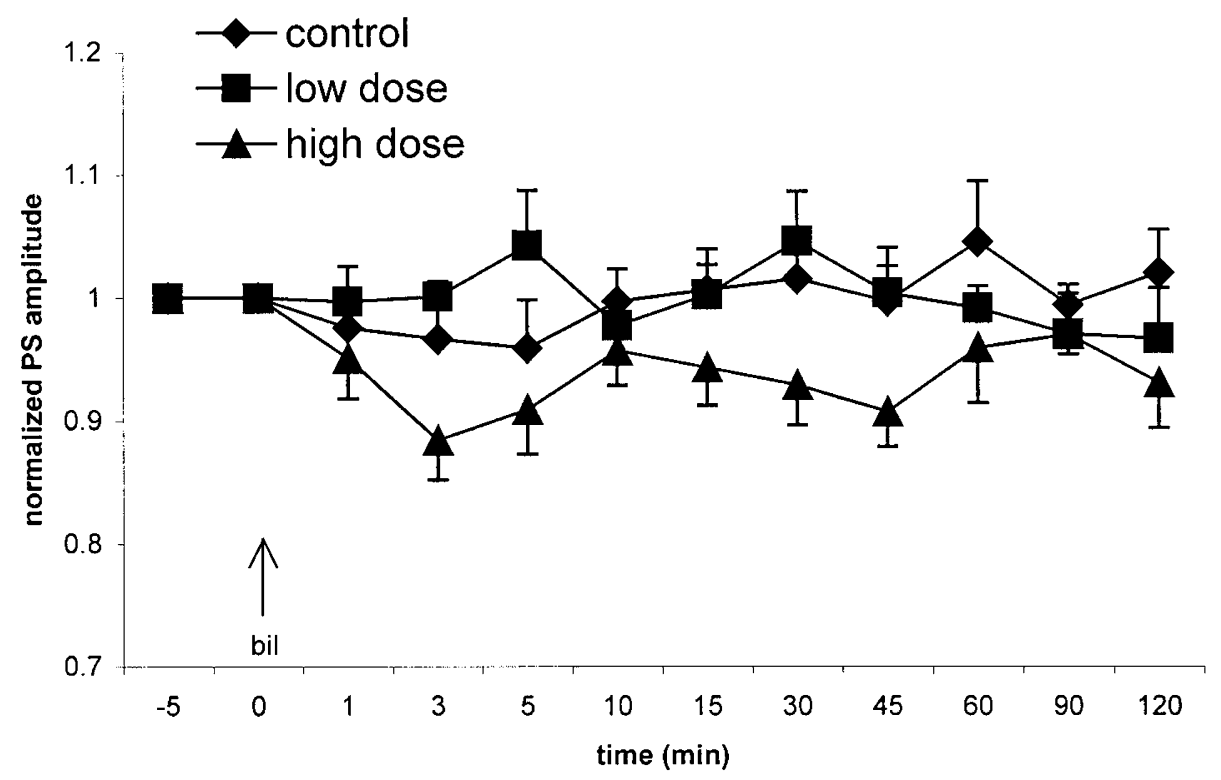

Figure 1. The effects of bilirubin on the normalized PS amplitude. The normalized PS amplitude is presented as the ratio of PS amplitude 5 min before and every time points after treatment. All numerical data are presented as mean \pm SEM, statistical significance was determined by ANOVA. The "bil" arrow indicates the starting time point of bilirubin application. The traces show the single recordings of PS at 5 min before and 60 min after bilirubin application (time point $-5 \mathrm{~min}$ and $60 \mathrm{~min})$. The three arrows indicate the initial PS images.

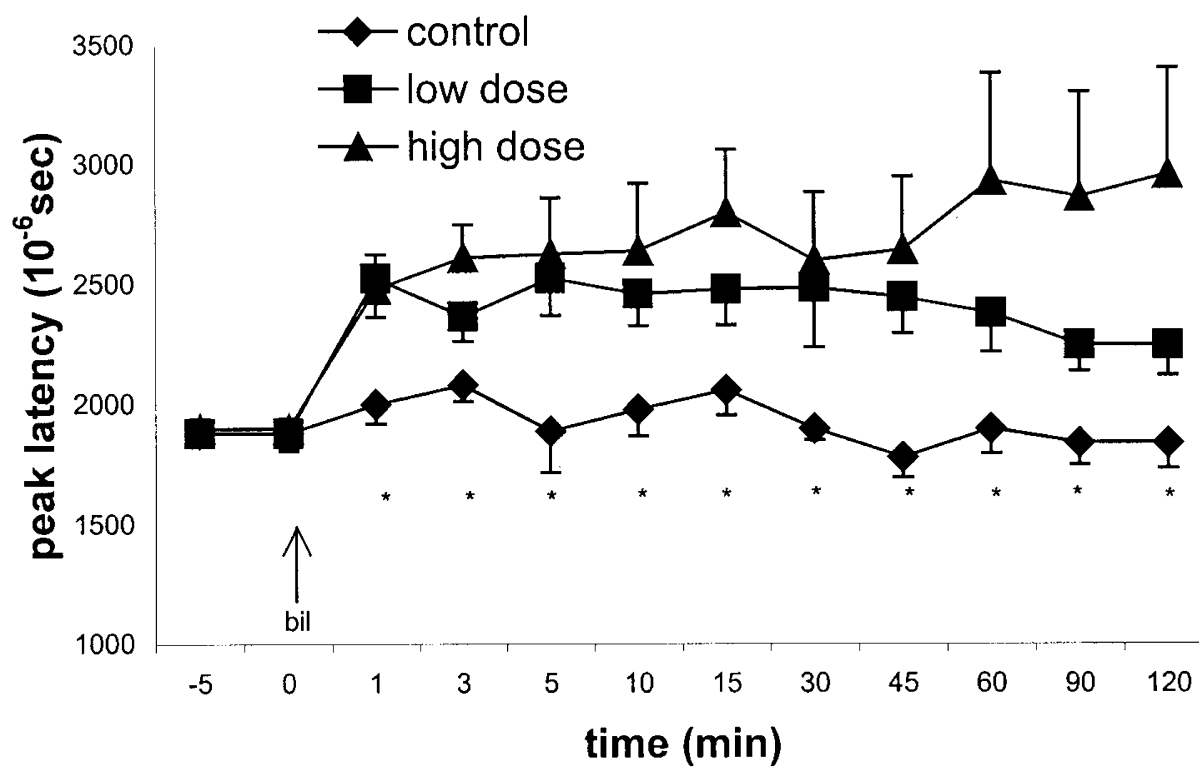

Figure 2. The effects of bilirubin on PS peak latency. Prolonged PS peak latencies were evident after low- or high-dose bilirubin treatment (bilirubin 30 and $60 \mathrm{mg} / \mathrm{kg})$ at all observed time points $(p<0.05)$, while the control group did not change. Both low- and high-dose bilirubin treated groups had longer PS peak latencies compared with the control group $(p<0.05)$. All numerical data are presented as mean \pm SEM, statistical significance was determined by ANOVA. The "bil" arrow indicates the starting time point of bilirubin application. * $p<0.05$, compared with preinjection and control group.

bilirubin-treated groups. Furthermore, the PS amplitudes of the two treated groups were smaller than that of the control group at each observed time point $(p<0.05$ at every point except $p$ $<0.01$ in the high-dose group at $3 \mathrm{~min}$ ). These results showed that bilirubin inhibited the induction of LTP in the $\mathrm{CA}_{3}$ region.

\section{DISCUSSION}

Herein we have shown that bilirubin prolongs the PS peak latency, decreases its slope and inhibits LTP formation following high-frequency stimulation in rat hippocampus in vivo. The 


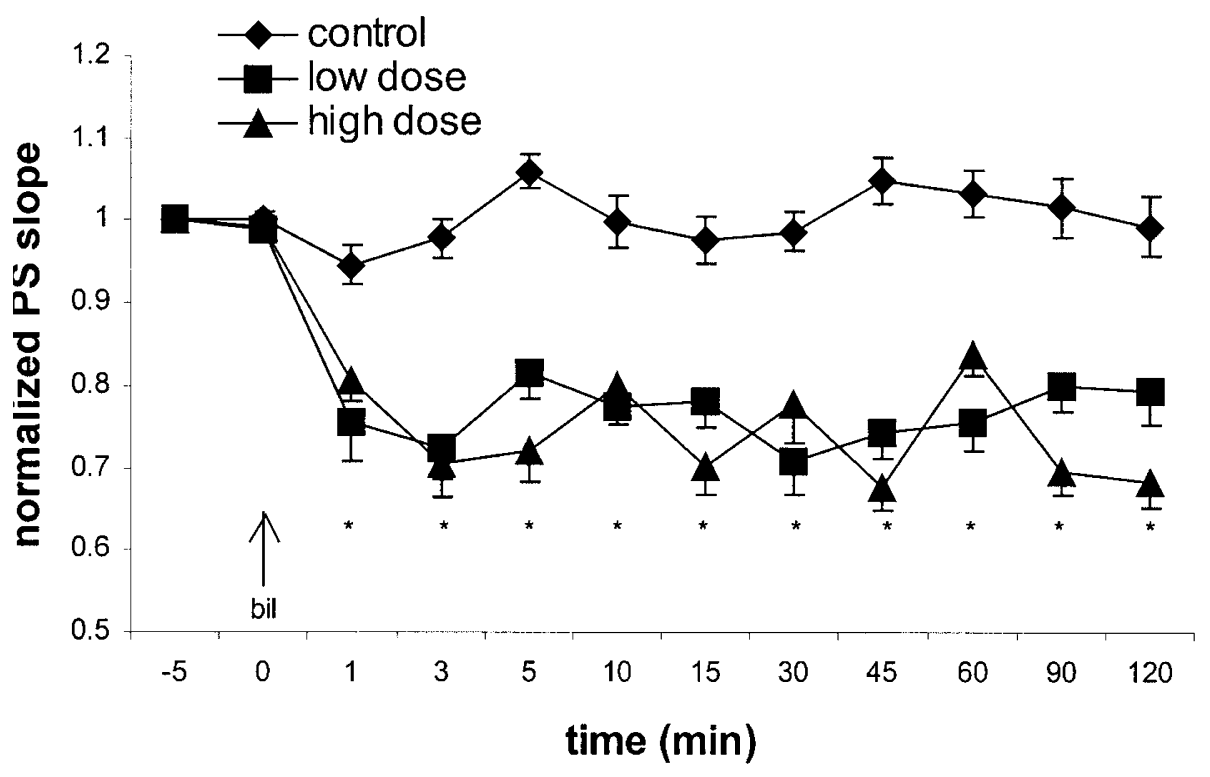

Figure 3. The effects of bilirubin on the normalized PS slope. Both bilirubin treated groups showed significant decrease of normalized PS slope $(p<0.05)$ at all observed time points. But no differences were found between the two groups. The normalized PS slope of control group stayed unchanged. All numerical data are presented as mean \pm SEM, statistical significance was determined by ANOVA. The "bil" arrow indicates the starting time point of bilirubin application. $* p<0.05$, compared with preinjection and control group.

first observation is compatible with previous findings in rat transverse hippocampal slices in vitro (6). To the best of our knowledge the observation regarding inhibition of LTP formation is novel. However, as LTP is thought to be the neurophysiologic foundation of learning and memory $(7,8)$, our observations appear to be compatible with previous findings of altered stimulus processing in rats previously subjected to short-term hyperbilirubinemia (9).

Bilirubin has been shown to enter the brain following i.v. administration (10-13). Thus, the experimental conditions used in our experiments were patterned on those previously shown to result in significant bilirubin concentrations in the brain. Under similar conditions the half-life of bilirubin in whole brain has been between $16.1 \pm 7.7$ (13) and $18 \pm 21.5$ min (mean \pm SD) (10). Similarly, the half-life in hippocampus has been $17.1 \pm 8.9$ (13) to $19.0 \pm 21.5 \mathrm{~min}$ (10). The observation time in our experiments was therefore equivalent to 6-7 half-lives, suggesting that there may be residual effects of bilirubin neurotoxicity at a time when only minimal amounts of bilirubin remain in the brain. Previous studies found that bilirubin can be detected in the brain at 15 min after administration $(10,13)$. Although the brain bilirubin concentrations have not been quantitatively measured in our study, we started our electrophysiological observation 1 min after bilirubin infusion and had actually noted significant effects on PS as well as on LTP. It seems likely that small amounts of bilirubin start to enter the brain shortly after infusion of bilirubin is begun, and well before the 15-min time point where brain bilirubin was measured in previous studies $(10,13)$.

In this context it is of interest to note that changes in auditory brainstem responses in jaundiced infants have shown normalization following treatment with exchange transfusions (14). Herein it seems that bilirubin induced effects can be maintained by very low brain concentrations and possibly even after bilirubin has been totally cleared from the brain. If the experimental animals could survive longer, it would be of interest to study whether these effects are reversible. Different aspects of neuronal function are measured by auditory brainstem response (ABR) and by hippocampal PS and LTP, and it appears possible that bilirubin neurotoxicity may affect individual neuronal functions in distinctive ways and with variable degrees of reversibility. We suspect that neither normalization of ABR nor serum bilirubin can completely guarantee the long-term prognosis of infants who have suffered from hyperbilirubinemia. Comprehensive and long-term evaluations of neuronal function are needed. The widespread inhibitory effect of bilirubin on protein/peptide phosphorylation reactions certainly seems compatible with this hypothesis (2).

Our results show that bilirubin can increase the PS peak latency and decrease its slope without changing its amplitude. Similar results have been published by Hansen et al. (6)who showed that concomitant with a significant decrease in the slope, exposure to bilirubin increased the peak latency of the PS and inhibited synaptic activation in rat transverse hippocampal slices in vitro. The smaller slope and longer PS peak latency are the signs of decreased excitability in the nervous system. One possible explanation for this effect might be bilirubin-induced impairment of neurotransmitter metabolism. One study suggested that bilirubin could affect the release of neurotransmitters by inhibiting the phosphorylation of synapsin I (3). Other researchers found that bilirubin could impair neurotransmitter synthesis by inhibiting the uptake of tyrosine (precursor of dopamine) (15). Several other studies have also been compatible with a bilirubin-associated impairment of neurotransmitter metabolism (16-18).

Bilirubin could also disturb the homeostasis of neuronal membranes. Mayor et al. (19) reported that $40 \mu \mathrm{M}$ bilirubin increased the permeability of synaptosomal membranes. Stud- 
control

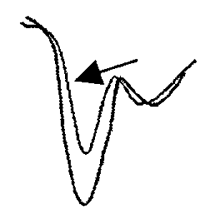

low dose

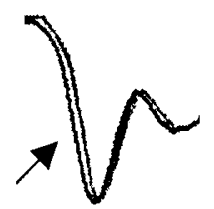

high dose

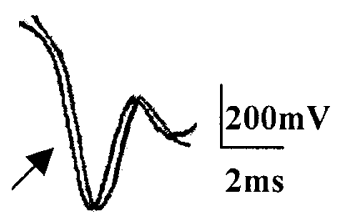

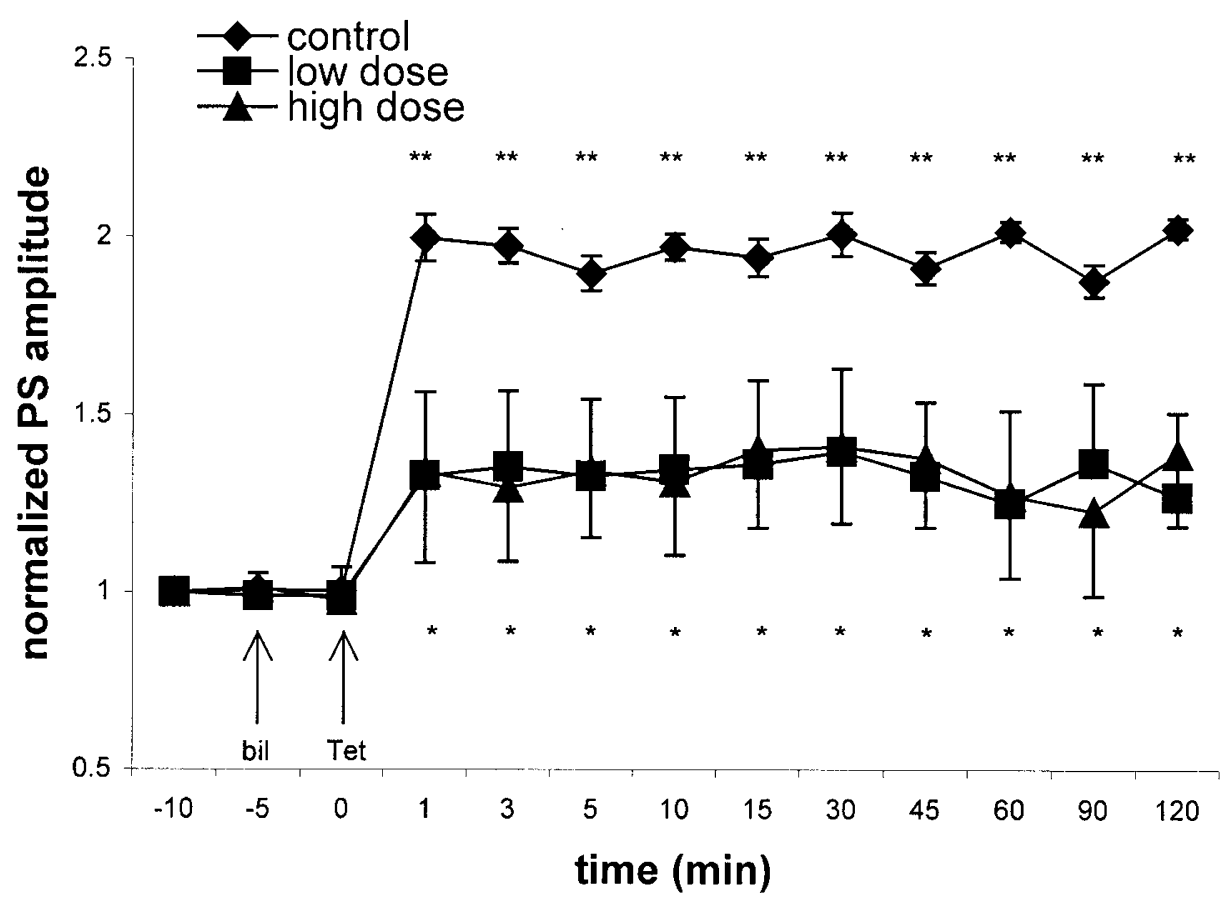

Figure 4. The effects of bilirubin on normalized PS amplitude after high-frequency stimulation. The PS amplitude of the control group was significantly increased after stimulation $(p<0.01)$. The PS amplitudes of the two treated groups were smaller than that of the control group at each observed time point $(p$ $<0.05$ ). All numerical data are presented as mean \pm SEM, statistical significance was determined by ANOVA. The "bil" arrow indicates the starting time point of bilirubin application, while the "Tet" arrow indicates the starting of tetanus stimulation. The traces show PS patterns at the time point - 10 and 60 min of the single animals. The arrows indicate the recordings 10 min before high frequency stimulation. ${ }^{*} p<0.05$, compared with control group, $* * p<0.01$, compared with preinjection.

ies by other groups are also compatible with a bilirubinmediated increase in the permeability of neuronal membranes $(20,21)$. However, these observation not withstanding, the mechanism by which bilirubin changes the PS slope and peak latency without affecting its amplitude remains an enigma.

The existence of long-term potentiation, a phenomenon first described in 1973 (4), suggested that the transmission efficiency of the synapse could change continuously under certain circumstances. This indicates that synaptic function can adapt. The plasticity of the nervous system can be considered as a basis for behavioral adaptability including learning and memory. As a model to describe the plasticity of the nervous system, LTP has attracted the attention of many neurologists for more than twenty years. LTP is generally accepted to be a neurophysiologic foundation of learning and memory $(7,8)$.

The mechanism of LTP induction has been extensively investigated (8). Two main factors in the induction of LTP are the frequency and strength of the high-frequency stimulation. Strength can increase the amplitude of evoked potential by a single stimulus. On the other hand, a certain frequency can promote the coordination of many afferent fibers. When the membrane potential of the postsynaptic membrane is depolarized to some degree, $\mathrm{Mg}^{2+}$ will be removed and the passage will be opened. After the combination of transmitters with NMDA, $\mathrm{Ca}^{2+}$ will flow into the neurons, and the ensuing cascade of biochemical reactions will change the characteristics of the membrane and form LTP.

Among those biochemical reactions, the protein-kinase system plays an important role. The protein-kinase system can be activated by $\mathrm{Ca}^{2+}$ and exert a function in the LTP-inducing process. In the meantime, the protein-kinase system is essential for maintaining the LTP by auto-phosphorylation. Recent in vitro studies have showed that bilirubin has extensive inhibitory effects on many protein kinases $(2,22)$. This might explain the finding of an inhibitory effect of bilirubin on the inducing process of LTP in our in vivo study. As we have discussed earlier, bilirubin could also inhibit LTP by reducing synaptic activity through its effects on neurotransmitter metabolism and neuronal membranes. The novel finding that bilirubin may inhibit the induction of LTP in the hippocampus by short-term exposure, raises the possibility that neonatal hyperbilirubinemia could have an impact on the infant's learning and 
memory. There is some experimental support for this speculation (9). To gain insight into the possible mechanism underlying our observations, it might be of interest to investigate the effect of bilirubin on the afferent volley and the excitability of the perforant path.

Although hyperbilirubinemia is common in the neonatal period, a detailed understanding of the nature and extent of the putative long-term effects of this phenomenon still eludes us. Clinicians can neither identify the sequelae with certainty (23), nor agree on a consistent regimen for treatment (24). This strongly suggests a need for further studies of bilirubin neurotoxicity. Bilirubin may inhibit specific functions of the nervous system for a period that possibly exceeds the actual presence of bilirubin in the brain. Our findings suggest a potential effect on mechanisms associated with learning and memory. We speculate that valuable information may be gained from a more thorough study of behavior, learning, and memory in infants with significant hyperbilirubinemia, both acutely and over a longer term.

Acknowledgment. We are grateful to Professor T.W.R. Hansen for his helpful discussion and critical reading of the manuscript.

\section{REFERENCES}

1. Dennery PA, Rhine WD, Stevenson DK 1995 Neonatal jaundice-What now? Clin Pediatr 34:103-107

2. Hansen TWR, Mathiesen SBW, Walaas SI 1996 Bilirubin has widespread inhibitory effects on protein phosphorylation. Pediatr Res 39:1072-1077

3. Hansen TWR, Bratlid D, Walaas SI 1988 Bilirubin decreases phosphorylation of synapsin I, a synaptic vesicle-associated neuronal phosphoprotein, in intact synaptosomes from rat cerebral cortex. Pediatr Res 23:219-223

4. Bliss TVP, Lomo T 1973 Long-lasting potentiation of synaptic transmission in the dentate area of the anaesthetized rabbit following stimulation of the perforant path. J Physiol 232:331-356
5. Bliss TVP, Collingridge GL 1993 A synaptic model of memory: long-term potentiation in the hippocampus. Nature 361:31-39

6. Hansen TWR, Paulsen O, Gjerstad L, Bratlid D 1988 Short-term exposure to bilirubin reduces synaptic activation in rat transverse hippocampal slices. Pediatr Res 23:453456

7. Berger TW 1984 Long-term potentiation of hippocampal synaptic transmission affects rates of behavioral learning. Science 224:627-630

8. Madison DV, Malenka RC, Nicoll RA 1991 Mechanism underlying long-term potentiation of synaptic transmission. Annu Rev Neurosci 14:379-397

9. Hansen TWR, Sagvolden T, Bratlid D 1987 Open field behavior of rats previously subjected to short-term hyperbilirubinemia with or without blood-brain barrier manipulations. Brain Res 242:26-36

10. Hansen TWR, Cashore WJ 1995 Rates of bilirubin clearance from rat brain regions. Biol Neonate 68:135-140

11. Hansen TWR, Bratlid D 1989 Cerebral blood volumes in young rats with and without in situ saline flushing of cerebral vasculature. Biol Neonate 6:15-21

12. Hansen TWR 1995 Acute entry of bilirubin into rat brain regions. Biol Neonate 67:203-207

13. Hansen TWR 1996 Bilirubin entry into and clearance from rat brain during hypercarbia and hyperosmolality. Pediatr Res 39:72-76

14. Nwaesei CG, Van Aerde J, Boyden M, Perlman M 1984 Changes in auditory brainstem responses in hyperbilirubinemic infants before and after exchange transfusion. Pediatrics 74:800-803

15. Amato M 1995 Mechanism of bilirubin toxicity. Eur J Pediatr 154(9 suppl 4):S54S59

16. Roseth S, Hansen TWR, Fonnum F, Walaas SI 1998 Bilirubin inhibits transport of neurotransmitters in synaptic vesicles. Pediatr Res 44:312-316

17. Hansen TWR, Mathiesen SB, Sefland I, Walaas SI 1999 Bilirubin inhibits $\mathrm{Ca}^{++}$ dependent release of norepinephrine from permeabilized nerve terminals. Neurochem Res 24:733-738

18. Cashore WJ, Kilguss NV 1989 Inhibition of synaptosomal tyrosine uptake by bilirubin. Pediatr Res 25:209A

19. Mayor FJ, Diez-Guerra J, Valdivieso F, Mayor F 1986 Effect of bilirubin on the membrane potential of rat brain synaptosomes. J Neurochem 47:363-369

20. Cowger ML 1971 Mechanism of bilirubin toxicity on tissue culture cells: Factors that affect toxicity, reversibility by albumin, and comparison with other respiratory poisons and surfactants. Biochem Med 5:1-16

21. O'Callaghan A, Duggan PF 1984 Possible biochemical basis for bilirubin neurotoxicity. Biochem Soc Trans 12:483

22. Churn SB, Delorenzo RJ, Shapiro SM 1995 Bilirubin induces a calcium-dependent inhibition of multifunctional $\mathrm{Ca}^{2+} /$ Calmodulin-dependent kinase II activity in vitro. Pediatr Res 38:949-954

23. Watchko JF The clinical sequelae of hyperbilirubinemia. In: Maisels MJ, Watchko JF, editors. Neonatal jaundice. London: Harwood Academic Publishers; 2000: 115-135

24. Hansen TWR 1996 Therapeutic approaches to neonatal jaundice: An international survey. Clin Pediatr 35:309-316 